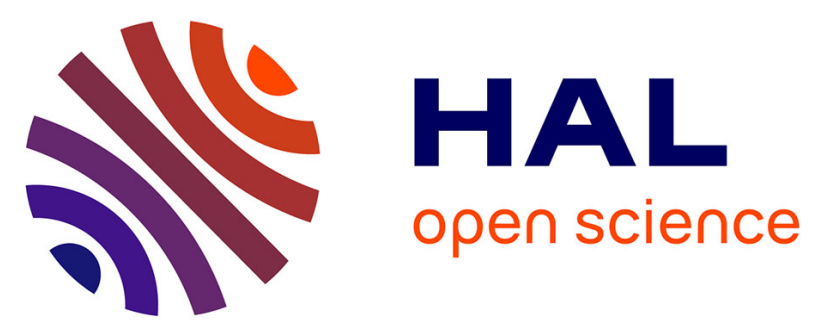

\title{
Integrating Diverse Reaction Types into Stochastic Models - A Signaling Pathway Case Study in the Imperative Pi-Calculus
}

Orianne Mazemondet, Mathias John, Carsten Maus, Stefan Leye, Adelinde Uhrmacher, Arndt Rolfs

\section{To cite this version:}

Orianne Mazemondet, Mathias John, Carsten Maus, Stefan Leye, Adelinde Uhrmacher, et al.. Integrating Diverse Reaction Types into Stochastic Models - A Signaling Pathway Case Study in the Imperative Pi-Calculus. Winter Simulation Conference (WSC) '09, Dec 2009, Austin, Texas, United States. 10.1109/WSC.2009.5429723 . hal-00656195

\section{HAL Id: hal-00656195 https://hal.inria.fr/hal-00656195}

Submitted on 3 Jan 2012

HAL is a multi-disciplinary open access archive for the deposit and dissemination of scientific research documents, whether they are published or not. The documents may come from teaching and research institutions in France or abroad, or from public or private research centers.
L'archive ouverte pluridisciplinaire HAL, est destinée au dépôt et à la diffusion de documents scientifiques de niveau recherche, publiés ou non, émanant des établissements d'enseignement et de recherche français ou étrangers, des laboratoires publics ou privés. 
Proceedings of the 2009 Winter Simulation Conference

M. D. Rossetti, R. R. Hill, B. Johansson, A. Dunkin, and R. G. Ingalls, eds.

\title{
INTEGRATING DIVERSE REACTION TYPES INTO STOCHASTIC MODELS - A SIGNALING PATHWAY CASE STUDY IN THE IMPERATIVE $\pi$-CALCULUS
}

\author{
Orianne Mazemondet \\ for Neuroregeneration (AKos) \\ University of Rostock \\ Gehlsheimerstr. 20 \\ 18147 Rostock, Germany
}

\author{
Mathias John \\ Carsten Maus \\ Adelinde M. Uhrmacher \\ Modelling and Simulation Group \\ Institute of Computer Science \\ University of Rostock \\ Albert-Einstein-Str. 21 \\ 18059 Rostock, Germany
}

Arndt Rolfs

\author{
Albrecht-Kossel-Institute \\ for Neuroregeneration (AKos) \\ University of Rostock \\ Gehlsheimerstr. 20 \\ 18147 Rostock, Germany
}

\begin{abstract}
We present a case study of reusing parameters and reactions of a deterministic model of a biochemical system in order to implement a stochastic one. Our investigations base on a model of the Wnt signaling pathway and aim to study the influence of the cell cycle on the pathway's dynamics. We report on our approaches to solve two major challenges: one is to gather and convert kinetic model parameters, e.g. constants for diffusion and enzymatic reactions. The second challenge is to provide the first implementation of reactions that exhibit Michaelis-Menten kinetics into a $\pi$-Calculus based approach by deploying the Imperative $\pi$-Calculus.
\end{abstract}

\section{INTRODUCTION}

Models in systems biology are hardly built from scratch, but typically deploy other models or model parts, e.g. Lecca et al. (2004) and Ciocchetta et al. (2008). This is facilitated by the rapidly increasing number of models published and partially stored in publicly accessible databases. However, this type of generating models also bears specific problems as fragments of models have to be combined with other sources of knowledge. Parameters and biochemical reactions are the prime subjects of this "white-box" reuse. Thereby, the modeler faces diverse problems, in particular when switching from the deterministic to the stochastic world and/or from one modeling formalism to another. In the following, a model of the Wnt/ $\beta$-catenin signaling pathway, which is being developed in our group, shall serve as a case study to illuminate some of these problems.

Investigating the mechanisms that influence the growth and development of human neural progenitor cells is of particular relevance for the study of e.g. Parkinson's or Alzheimer's diseases. A main focus of research in this field is on the $\mathrm{Wnt} / \beta$-catenin signaling pathway, an intracellular reaction network with decisive impact on the development of cells, e.g. neural stem cells (Lange et al. 2006). It has been subject of modeling efforts in other biological systems before, e.g. Lee et al. (2003), Tymchyshyn and Kwiatkowska (2008), and Cho et al. (2006). The goal of our simulation study is to analyze the characteristics of noise induced by the cell cycle, which is a sequence of states proliferating cells traverse, and their impact on the Wnt signaling pathway activity. Therefore, our model integrates information about the cell cycle into a Wnt signaling pathway model. The model is primarily based on Lee et al. (2003), which is a deterministic, continuous model, and adopts parameters from Tymchyshyn and Kwiatkowska (2008). We simplify the model by omitting reactions that we assume to be of less interest for our investigations. As recent studies reveal the importance to distinguish between activities in the nucleus and the cytosol of the cell (Willert and Jones 2006), we extend the original model by introducing compartments.

Furthermore, as the number of one of the key players, i.e. the degradation complex, is rather low, it appears suitable to move from a deterministic to a stochastic approach (Wolkenhauer et al. 2004). The resulting model comprises different reaction types like diffusion, Mass Action, and Michaelis-Menten, that need to be taken into account.

In the following, the developed model shall serve as a case study to address two challenges of building cell biological models by partial reuse. The first challenge is to gather model parameters and convert kinetic parameters into stochastic ones. We will discuss the various sources for biological data coming from both literature and wet-lab experiments and their use in the model. Therefore, we extend the work presented in Kuttler and Niehren (2006), where the integration of initial 
concentrations and association constants into stochastic modeling is presented and show how to obtain stochastic constants for diffusion reactions and Michaelis-Menten kinetics. The second challenge is on the side of the modeling language. We chose to implement our model in the Imperative $\pi$-Calculus (John et al. 2008, John et al. 2009), since we aim to explore its modeling power regarding the inclusion of different sorts of reactions and also reaction kinetics. The particular challenge here is to include reactions following Michaelis-Menten dynamics. Our result is, to the best of our knowledge, the first implementation of reactions with Michaelis-Menten kinetics in a $\pi$-Calculus based approach.

The paper is structured as follows. First we describe the Wnt signaling pathway and its key players and present the overall structure of our model in Section 2. We then present our solutions for gathering model parameters, including the conversion of kinetic parameters into stochastic ones in Section 3. Section 4 provides an introduction to the Imperative $\pi$-Calculus and in Section 5 an extract of the model, i.e. the implementation of reactions with Michaelis-Menten kinetics in the Imperative $\pi$-Calculus, is shown.

\section{THE WNT SIGNALING PATHWAY}

The main outcome of the $\mathrm{Wnt} / \beta$-catenin signaling pathway is an increase of the amount of $\beta$-catenin in response to Wnt molecules binding to receptors at the outside of the plasma membrane. In the absence of Wnt, a degradation complex is operating efficiently that reduces the amount of $\beta$-catenin. At the arrival of Wnt molecules, the degradation complex gets deactivated and consequently, due to its constant production, the amount of $\beta$-catenin increases. This affects the regulation and transcription of genes and accompanies the cells in their specialization (differentiation) process. We work in vitro with human neural progenitor cells (hNPCs) (Pollock et al. 2006). HNPCs start their differentiation asynchronously into neural cell types like e.g. astrocytes and neurons. This is due to their commitment to the cell cycle.

The cell cycle is a central biological process for cell and cell population growths. It is divided in four phases called G1, S, G2 and M. During the first one, G1, a cell has two possibilities: either to enter the cycle and to proceed with the three other phases or to start differentiation. Only cells in G1 are sensitive to the Wnt signal. In this way, the cell cycle interferes experimental studies on the activity of the $\mathrm{Wnt} / \beta$-catenin pathway. Thus, the question arises, whether observed patterns in wet-lab data can be explained under consideration of the cell cycle. Our model shall help to address this question.

We model the $\mathrm{Wnt} / \beta$-catenin signaling pathway during the differentiation of a single human neural progenitor cell. As shown in Figure 1, the cell contains two compartments: the cytosol and the nucleus, represented by two concentric spheres. The main actors of our model are $\beta$-catenin, Axin and the T-cell factor (TCF). In the cytosol, de-/phosphorylated Axin (Axin/AxinP) and $\beta$-catenin (BcatCyt) are located. The nucleus contains TCF and $\beta$-catenin (BcatNuc). Two reactions are defined for Axin: aDec, describing the decay of Axin, i.e. its number is simply reduced by one, and aPho representing its phosphorylation, i.e. Axin turns into AxinP. Symmetrically, the two reactions aDep and apDec represent the dephosphorylation and the decay of AxinP. Notice, that $a D e p$ occurs only in the presence of the Wnt signal. The activation of $a D e p$ is the only effect the Wnt signal has in our model. Reactions bProd and bDec are defined for BcatCyt, describing its production, i.e. its amount is increased by one, and its decay, respectively. The key reaction of the pathway is $b D e g$, which describes the AxinP-mediated degradation of BcatCyt. We assume bDeg to be an enzymatic reaction with AxinP being the enzyme and BcatCyt the substrate. Similarly, the production of Axin, aProd, involves BcatNuc as a substrate and TCF as an enzyme. Reactions bIn and bOut represent the translocation of $\beta$-catenin from the cytosol to the nucleus and back. The cell cycle is abstracted by two reactions, $p h S$ and $p h G 2$, that introduce the activation delay of the signaling pathway. Once reaction $p h G 2$ is performed, aDep, i.e. the dephosphorylation of AxinP, is enabled immediately. Except bDeg and $a$ Prod, which follow Michaelis-Menten kinetics, we assume Mass Action kinetics for all reactions in the model.

Our model is an adaptation of the work by Lee et al. (2003) from which we made the following simplifications: We reduced the pathway components to the three major proteins mentioned above. The binding of Wnt molecules to receptors and the signal transduction at the cell membrane are abstracted as these processes still remain poorly defined and understood. The pathway activation was originally described by a time-dependent function. In our model activation is a discrete step, immediately performed after achievement of the cell cycle. We simplified the composition of the degradation complex by only referring to Axin as its unique component. This is possible due to the low amount of Axin available in the cell compared to the other components of the degradation complex. This last assumption reinforced the suitability of a stochastic model.

\section{STOCHASTIC PARAMETERIZATION}

In this section, we show how we calculated the different sorts of parameters needed for our model. In Table 1, we present the parameters as they are used in our model, report source of the data, and refer to the approach used to calculate them. 


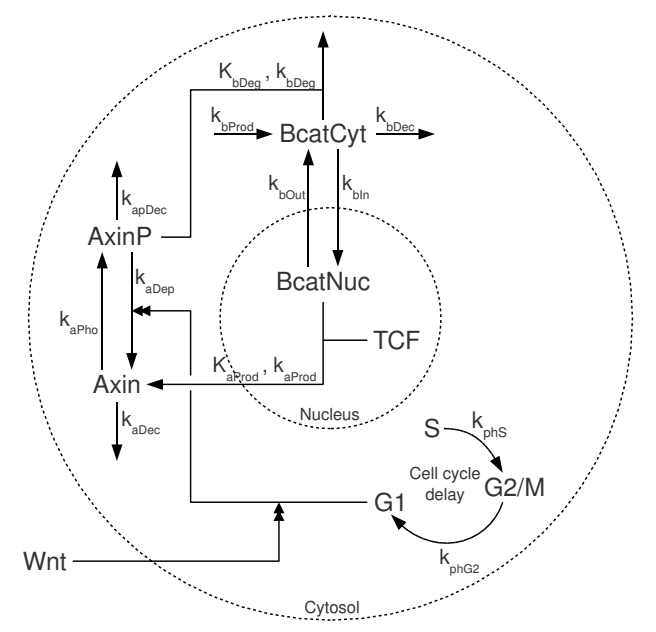

Figure 1: Schematic overview of the model. Double-headed arrows indicate that reactants influence a process but are not consumed. Reaction rate constants begin with a lower case $k$ and dissociation constants with a capital $K$ and correspond with the parameter names given in Table 1. The two compartments, i.e. cytosol and nucleus, are indicated by dashed circles.

\section{(I) Compartment volumes.}

The volume is a prerequisite for calculating stochastic parameters as the reaction speed is dependent on the probability of molecules to collide. Unfortunately, in many ordinary differential equation (ODE) models data on the volume is not reported. Thus, as no information in the literature could be found, we measured the volume of hNPCs and their cytosol and nucleus using microscopy and impedance as a control.

\section{(II) Molecules' number and stochastic rate constants.}

Essential parameters of stochastic models are the initial number of molecules and stochastic rate constants. As described in Kuttler and Niehren (2006), they are obtained from initial concentrations and kinetic rate constants. Concentrations are usually given in $\mathrm{mol} / \mathrm{l}$ (sometimes also denoted by $M$ ), i.e. amount of molecules per volume. Thus, in order to obtain number of molecules $N$, concentrations are multiplied by the Avogadro constant $\left(N_{A} \approx 6.023 \cdot 10^{23} \mathrm{~mol}^{-1}\right)$, i.e. number of molecules per mole, and by the volume of the compartment the species resides in:

$$
N=C \cdot N_{A} \cdot V
$$

where $C$ is the concentration and $V$ is the volume of the cell or compartment.

The stochastic rate constant $k_{\text {sto }}^{0}$ of zero order reactions, i.e. reactions without reactants, is calculated based on the volume, the Avogadro constant, and the kinetic rate constant $k$ :

$$
k_{s t o}^{0}=k \cdot N_{A} \cdot V
$$

For first order reactions, the kinetic and the stochastic rate constant coincide. For second order reactions, i.e. reactions with two reactants, the stochastic rate constant $k_{s t o}^{2}$ is calculated by:

$$
k_{\text {sto }}^{2}=\frac{k}{N_{A} \cdot V}
$$

\section{(III) Diffusion rate constants.}

We follow the approach in Elf and Ehrenberg (2004), i.e. we consider compartments to be separated containers, called sub-volumes, between which molecules diffuse in a discrete event manner. A diffusion event describes the motion of one particle from the center of one compartment to the center of the other. Thereby, the cytosol and the nucleus form a structure of two concentric spheres, the first surrounding the latter, see Figure 1. To determine the rate constants of diffusion events 
Table 1: Stochastic model parameters. Description gives information about the reaction, values contains the calculated value as used in the model, calculation refers to the way the value is calculated, and source provides the source of the data the calculation is based on: [1] Lee et al. (2003), [2] Krieghoff (2006), [3] Alam et al. (2004), or [4] Schaeffer (2008).

\begin{tabular}{|c|c|c|c|}
\hline Description & Values & Calculation & Source \\
\hline Initial amount of Axin molecules & NAxin $=0$ & I, II & [1] \\
\hline Initial amount of phosphorylated Axin molecules & $N A x i n P=11$ & I, II & [1] \\
\hline Initial cytosolic amount of $\beta$-catenin molecules & NBcatCyt $=18390$ & I, II & [1] \\
\hline Initial nuclear amount of $\beta$-catenin molecules & NBcatNuc $=7483$ & I, II & {$[1]$} \\
\hline Initial amount of TCF molecules & $N T C F=3207$ & I, II & [1] \\
\hline$\beta$-catenin production rate constant & $k_{b P r o d}=222.3 \mathrm{~min}^{-1}$ & I, II & [1] \\
\hline$\beta$-catenin decay rate constant & $k_{b D e c}=2.57 \cdot 10^{-4} \mathrm{~min}^{-1}$ & I, II & [1] \\
\hline$\beta$-catenin diffusion rate constant into the cytosol & $k_{\text {bOut }}=1.65 \cdot 10^{-5} \mathrm{~min}^{-1}$ & I, III & [2] \\
\hline$\beta$-catenin diffusion rate constant into the nucleus & $k_{b I n}=6.72 \cdot 10^{-6} \mathrm{~min}^{-1}$ & I, III & [2] \\
\hline S phase delay & $k_{p h S}=3.06 \cdot 10^{-3} \mathrm{~min}^{-1}$ & IV & [3] \\
\hline G2/M phase delay & $k_{p h G 2}=6.12 \cdot 10^{-3} \mathrm{~min}^{-1}$ & IV & [3] \\
\hline$\beta$-cat. degradation (Michaelis-Menten parameter) & $K_{b D e g}=63052, k_{b D e g}=206 \mathrm{~min}^{-1}$ & I, II, V & [1] \\
\hline Axin production (Michaelis-Menten parameter) & $K_{a \text { Prod }}=6414, k_{\text {aProd }}=0.043 \mathrm{~min}^{-1}$ & I, II, V & [1] \\
\hline Axin decay & $k_{a D e c}=0.005 \mathrm{~min}^{-1}$ & I, II & [4] \\
\hline Phosphorylated Axin decay & $k_{a p D e c}=0.002 \mathrm{~min}^{-1}$ & I, II & [4] \\
\hline Axin phosphorylation & $k_{a P h o}=0.2 \mathrm{~min}^{-1}$ & I, II & [4] \\
\hline Axin dephosphorylation & $k_{a D e p}=0.1 \mathrm{~min}^{-1}$ & I, II & [4] \\
\hline
\end{tabular}

(diffusion constants), we start off with Fick's first law

$$
J=-D \frac{d \phi}{d x}
$$

that describes the diffusive flux $J$ in $\left[\mathrm{mol} \cdot \mathrm{m}^{-2} \cdot \mathrm{min}^{-1}\right]$ through the unit area. It depends on the diffusivity $D$ of the diffusing species in $\left[\mathrm{m}^{2} \cdot \mathrm{s}\right]$ and the concentration gradient $d \phi / d x$ in $\left[\mathrm{mol} \cdot \mathrm{m}^{-4}\right]$, with the direction of the latter being against the motion. We are interested in the rate constant of a single particle moving, such that we consider a difference of one particle between the two compartment centers. Therefore, we set the gradient to $d \phi / d x=-1 / x$, where $x$ is the absolute distance between the compartment centers. Notice, that by this step, we convert the gradient from a concentration difference over some distance with unit $\left[\mathrm{mol} \cdot \mathrm{m}^{-4}\right]$ into a difference of particle numbers over some distance with unit $\left[\mathrm{m}^{-1}\right]$. Since $J$ is given for the unit area, we need to multiply by the area $A$ connecting the cytosol and the nucleus. Under consideration of the volume $V$ of the compartment where the molecule starts, we obtain the stochastic diffusion constant

$$
D_{s t o}=\frac{D \cdot A}{x \cdot V}
$$

giving us the intended unit $s^{-1}$ in its final result. Notice, however, that in our model we do not use the time unit $s$ but $\min$, such that further conversions are required. We obtain the values for this formula in the following way. $D$ can be calculated based on a half-recovery time (HRT), which is experimentally determined by a technique called Fluorescence Recovery After Photobleaching (FRAP), from $D=\left(w^{2}\right) /(4 \cdot H R T)$, where $w$ is the width of the laser beam used for bleaching. As presented in Krieghoff (2006), the half-recovery time of $\beta$-catenin is $H R T_{\beta}=2: 12$ min with a width of the laser beam $w=514 \cdot 10^{-9} \mathrm{~m}$. The diffusion surface $A$ is given by the average amount and the radius of pores covering the nucleus, as presented in Panté and Kann (2002) $A=3.58 \cdot 10^{-12} \mathrm{~m}^{2}$. The diffusion distance $x$ results from our compartment model with two concentric spheres, $x=r_{N u c}+\left(r_{C y t}-r_{N u c}\right) / 2$, where $r_{X}$ is the radius of the sphere bordering compartment $X$.

\section{(IV) Cell cycle delay.}

In our model, the cell cycle induces a delay on the activation of the Wnt signaling pathway. Based on Smith and Martin (1973), we assume that the progression of a cell from one cell cycle phase to the next one follows exponential distribution. 
Thus, we need to obtain (1) the probability of a cell to start in a specific phase and (2) the rate constant for it to proceed. We determined (1) by performing Fluorescence-activated cell sorting analysis (FACS). (2) yields the inverse of the phase's duration, which is according to Alam et al. (2004), for G1 50\%, for S 33.3\%, and for G2/M 16.7\% of the entire duration of the cell cycle. The cell cycle duration of hNPCs was obtained experimentally.

\section{(V) Michaelis-Menten kinetics.}

A typical enzymatic reaction can be divided into a reversible binding of the substrate, which leads to an intermediate enzyme-substrate complex, and the actual enzymatic reaction step:

$$
E+S \underset{k_{-1}}{\stackrel{k_{1}}{\longrightarrow}} E S \stackrel{k_{2}}{\longrightarrow} E+P
$$

with $k_{1}$ as the rate constant of the association (forward) and $k_{-1}$ of the dissociation (backward) reaction of the substrate binding, and $k_{2}$ as the reaction rate constant of the irreversible enzymatic step. Enzymes $E$ are molecules acting as catalysts, i.e. they facilitate the reaction of a substrate $S$ to a product $P$ without their own consumption. Many enzymatic reactions take place with relatively low amounts of enzyme molecules compared to the substrate amounts and follow Michaelis-Menten kinetics. This allows for omitting the detailed Mass Action kinetics (6) and apply a more abstract equation for describing the reversible binding and enzymatic reaction in a single step. The Michaelis-Menten theory describes the kinetics of enzyme reactions as a hyperbolic saturation curve where even at very high substrate concentrations the maximum reaction speed is limited by substrate saturation of the enzyme. The maximum speed of the reaction $v_{\max }$ therefore depends strongly on the total enzyme amount: $v_{\max }=k_{2} \cdot[E]$. The substrate concentration, at which the reaction rate reaches half of its maximum speed is called the Michaelis constant $K_{M}$ and is defined as follows: $K_{M}=\left(k_{-1}+k_{2}\right) / k_{1}$. In the most simple case, when the final enzymatic step is the rate-limiting step, i.e. $k_{2}$ is much lower than $k_{-1}$, the Michaelis constant is almost equal to the dissociation constant of the enzyme-substrate complex:

$$
K_{M} \approx \frac{k_{-1}}{k_{1}}=K_{d}, \text { if } k_{2} \ll k_{-1}
$$

The dissociation constant $K_{d}$ specifies the propensity of the complex to separate into its smaller parts, i.e. the ratio between the association (forward) and dissociation (backward) reaction. Applying the approximation of $K_{M} \approx K_{d}$ can be very helpful as both rate constants for the binding $\left(k_{1}\right)$ and unbinding $\left(k_{-1}\right)$ reactions are, unlike to the dissociation constant, typically hard to derive from wet-lab experiments. All together, i.e. the substrate amount $[S]$, the maximum reaction rate $v_{\max }$, and $K_{M}$, determine the reaction rate $v$ of the whole enzymatic reaction described by the Michaelis-Menten equation:

$$
v=\frac{v_{\max } \cdot[S]}{K_{M}+[S]} \approx \frac{k_{2} \cdot[E] \cdot[S]}{K_{d}+[S]}
$$

For example, for Axin-mediated $\beta$-catenin degradation the rate equation yields

$$
v \approx \frac{k_{b D e g} \cdot[\text { Axin } P] \cdot[\text { BcatCyt }]}{K_{b D e g}+[\text { BcatCyt }]} .
$$

For stochastic simulation, the parameters for the Michaelis-Menten equation have to be parameterized according to the methods described above. The differences between detailed Mass Action and more abstract Michaelis-Menten kinetics for modeling enzymatic reactions are also nicely described in Breitling et al. (2008).

\section{THE IMPERATIVE $\pi$-CALCULUS}

Conceptually, the Imperative $\pi$-Calculus pursuits two main ideas: First, it allows to associate reactants with attributes, describing e.g. their internal state or their location in space. Reaction constraints are assigned to reaction rules, possibly taking the attribute values of reactants into account. E.g., the de-/phosphorylation of Axin in our model of the Wnt signaling pathway can be implemented by introducing a reactant $\operatorname{Axin}(\mathrm{S})$ with one attribute, representing the two possible states Axin('free') and Axin('phos'). The fact that Axin(s) can only be dephosphorylated in its phosphorylated state 
and vice versa can be expressed by the two reaction rules

$$
\text { Axin('free') } \stackrel{k_{\text {pho }}}{\longrightarrow} \text { Axin('phos') Axin('phos') } \stackrel{k_{d e p}}{\longrightarrow} \text { Axin('free'), }
$$

where $k_{d e p}$ and $k_{p h o}$ are the rate constants of Axin de-/phosphorylation, respectively. Notice, that throughout the paper, we freely use names in our implementations that directly refer to the parameters given in Table 1 . The reactions rules above implicitly include simple constraints on attribute values, since they can only be applied to either state Axin (' $\left.f r e e^{\prime}\right)$ or Axin ('phos'). More explicitly this idea is illustrated by the rules

$$
\operatorname{Axin}(\mathrm{s}) \stackrel{\text { if } \mathrm{s}=^{\prime} \text { free' then } k_{\text {pho }} \text { else } 0}{\longrightarrow} \operatorname{Axin}\left({ }^{\prime} \mathrm{phos}^{\prime}\right) \quad \operatorname{Axin}(\mathrm{s}) \stackrel{\text { if } \mathrm{s}^{\prime} \mathrm{phos}^{\prime} \text { then } k_{\text {dep }} \text { else } 0}{\longrightarrow} \text { Axin('free'), }
$$

where the rate constants upon the arrows are replaced by explicit reaction constraints. Here the reaction constraints directly define rate constants, possible in dependence of the reactants' attributes. In this sense they can also be seen as functional rate constants. Notice, that a rate constant of 0 ensures that a reaction never occurs even if the reactants are available. A more compact form of the rules above is obtained by writing

$$
\operatorname{Axin}(\mathrm{s}) \stackrel{\text { if } \mathrm{s}^{\prime} \mathrm{frree}^{\prime} \text { then } k_{\text {pho }} \text { else } k_{d e p}}{\longrightarrow} \operatorname{Axin}\left(\text { if } \mathrm{s}={ }^{\prime}\right. \text { free' then 'phos' else 'free'), }
$$

provided that the attribute values of reactants can be described by expressions like if-then-else. In the following, we stick to this form as it is closest to the actual description in the Imperative $\pi$-Calculus.

The second idea is to introduce a global store which maps names to values. Constraints can contain assignments that change the mapping, thus, introducing side effects. Notice, that value changes are not committed to the global store until a reaction is actually performed. Thus, constraints do not influence each other during evaluation. The effects of assignments are taken into account by constraint (re-)evaluation before every reaction. E.g., in our model, the dephosphorylation of Axin can only be performed if the Wnt pathway is activated. As described in Section 2, the Wnt signal is on immediately when the cell cycle enters state G1. This can be implemented by

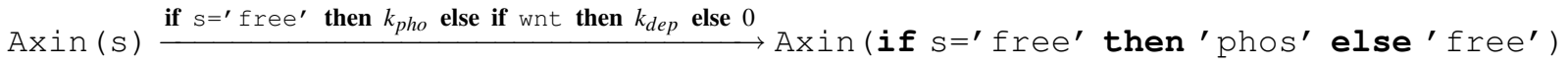

$$
\begin{aligned}
& \text { Cycle (s) } \stackrel{\text { if } s={ }^{\prime} G 2^{\prime} \text { then wnt: }:=\text { true; } k_{p h G 2} \text { else } 0}{\longrightarrow} \text { Cycle ('G1'), }
\end{aligned}
$$

where $k_{p h G 2}$ is the rate constant for the cell cycle switching from state G2/M to state G1 and true is a boolean constant. The presence of the Wnt signal is captured by the global name wnt. We introduce a rather abstract reactant Cycle (s), with possible states Cycle $\left({ }^{\prime} G 1^{\prime}\right)$ and Cycle $\left({ }^{\prime} G 2^{\prime}\right)$. For simplicity, state Cycle $\left({ }^{\prime} S^{\prime}\right)$ is omitted in this example. When Cycle ('G2') turns into Cycle $\left({ }^{\prime} \mathrm{G} 1^{\prime}\right)$ the value true is assigned to the global name wnt. By extending the constraint of the Axin dephosphorylation rule it is ensured, that the reaction can only occur when the Wnt signal is on, i.e. when wht $=$ true. Figure 2 shows a possible evolution of a system with one Cycle ('G2'), one Axin ('free' ), two Axin('phos'), and wht being set to the boolean constant false. Initially, our reaction rules define four reactions, one for each reactant in the system. However, reactions turning Axin ('phos') into Axin ('free') can never occur, since their reaction constraint evaluates to 0 for wht $=$ false. When $C y c l e\left({ }^{\prime} G 2^{\prime}\right)$ turns into $C y c l e\left({ }^{\prime} G 1^{\prime}\right)$, the mapping of wht in the global store is changed to true. Because of constraint reevaluation the rate constant of all reactions from Axin ('phos') to Axin ('free') changes to $k_{d e p}$. Since we did not define any reaction rule involving Cycle $\left({ }^{\prime} G 1^{\prime}\right)$ as a reactant, the number of potential reactions reduces to 3 .

In the remainder of this section, we first introduce the $\pi$-Calculus and then describe the attribute language, which is used to express both attribute values and reaction constraints. Thereupon, we show in which way the Imperative $\pi$-Calculus extends the $\pi$-Calculus. Due to spatial restrictions and since we do not wish to prove the correctness of our encodings in Section 5, we omit formal definitions of semantics. We prefer to provide informal descriptions and examples in order to support the understanding of the basic ideas and mechanisms of the language. For a complete formal definition of the Imperative $\pi$-Calculus see (John et al. 2009) and (John et al. 2008).

The $\pi$-Calculus. The $\pi$-Calculus (Milner 1999) defines infinite sets of process names Proc and channel names Chans. The syntax of the $\pi$-Calculus is shown in Figure 3. Processes can communicate on channels, i.e. they can send $x ! \tilde{y}$ and receive $x ? \tilde{y}$. Thereby, $x$ defines the subject of the communication, i.e. the channel the communication is performed on, and $\tilde{y}$ the 
Mazemondet, John, Maus, Uhrmacher and Rolfs

\begin{tabular}{|c|c|c|c|}
\hline \begin{tabular}{|l} 
wnt \\
false
\end{tabular} & \begin{tabular}{|l} 
Wnt \\
false
\end{tabular} & $\longleftarrow$ & $\begin{array}{c}\text { wnt } \\
\text { true }\end{array}$ \\
\hline Axin('free') $\stackrel{k_{p h o}}{\longrightarrow}$ Axin ('phos') & \multirow{4}{*}{\multicolumn{2}{|c|}{$\stackrel{\text { Cycle ('G2') } \longrightarrow \text { Cycle ('G1') }}{\longrightarrow}$}} & Axin('free') $\stackrel{k_{p h o}}{\longrightarrow}$ Axin ('phos') \\
\hline Cycle('G2') $\stackrel{k_{G 2}}{\longrightarrow} \mathrm{Cycle}(' \mathrm{G1}$ ') & & & Axin('phos') $\stackrel{k_{\text {dep }}}{\longrightarrow} \operatorname{Axin}($ 'free') \\
\hline Axin('phos') $\stackrel{0}{\longrightarrow} \operatorname{Axin}(' f r e e ')$ & & & Axin('phos') $\stackrel{k_{\text {dep }}}{\longrightarrow} \operatorname{Axin}($ 'free') \\
\hline Axin('phos') $\stackrel{0}{\longrightarrow} \operatorname{Axin}('$ free') & & & \\
\hline
\end{tabular}

Figure 2: A possible evolution of a system with one Cycle('G2'), one Axin ('free'), two Axin ('phos'), and wnt $=$ false. Reactions that can occur are highlighted with a gray background. By changing the value of wnt from false to true, the rate constants of all reactions from Axin('phos') to Axin('free') change from 0 to $k_{d e p}$.

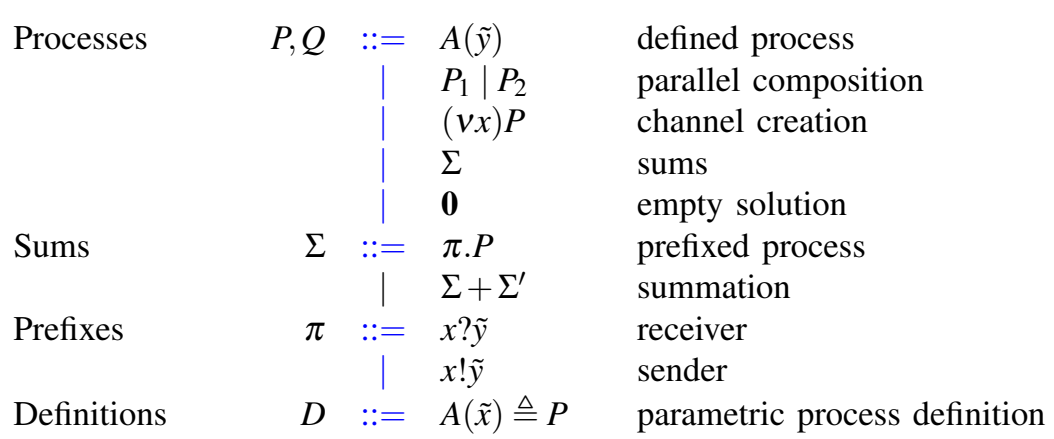

Figure 3: Syntax of the $\pi$-Calculus: $x, \tilde{x}, \tilde{y} \in$ Chans

objects of the communication, i.e. those channels which are send or received. With $(v x) P$ a new channel is introduced with scope on $P . P_{1} \mid P_{2}$ denotes concurrently running processes and + the exclusive choice between different communications a process can perform. The $\mathbf{0}$ process does nothing and is usually omitted. Process definitions $A(\tilde{x}) \triangleq P$, with $A \in \operatorname{Proc}$, allow to define processes with parameters. They are instantiated by defined processes $A(\tilde{y})$ that call definition $A(\tilde{x})$ with a specific tuple of channels. Whenever we write $A\left(y_{1} \ldots y_{n}\right)$, we assume that a process definition $A\left(x_{1} \ldots x_{n}\right) \triangleq P$ is declared. No two processes shall be defined with the same name. Notice, that process definitions already support the basic concept of attributes. E.g., we could introduce channel names phos and free and instantiate Axin(s) by Axin (phos) and Axin (free). However, without reaction constraints, the use of this concept is limited, such that we prefer distinct process definitions for different states, e.g. Axin() and AxinP().

Following the ideas in Regev and Shapiro (2004), $\pi$-Calculus programs that describe reaction networks consist of a set of process definitions and one initial process. The process definitions introduce the reacting species, e.g. Axin () and AxinP (). The initial process (or initial solution) is a parallel composition of defined processes that describes the molecules, i.e. the species instances, which are initially in the system. For example, a model of Axin de-/phosphorylation not considering the cell cycle and an initial solution of $5 \mathrm{Axin}()$ and $5 \mathrm{AxinP}()$ is shown in Figure 4, where $\prod_{i=1}^{n} P$ abbreviates a parallel composition of $n$ processes. In the $\pi$-Calculus, each communication needs to have exactly one sender and one receiver. On one hand this means that no reactions can be written with more than two reactants. Although, this reflects the basic assumption for the stochastic modeling of biochemistry (Gillespie 1976), it also shows a basic limitation of the $\pi$-Calculus compared to other formalism like BioPEPA (Ciocchetta and Hillston 2008), Petri-Nets (Heiner et al. 2008), or the BioNetGen (Danos et al. 2007). However, with the help of the imperative extension it might be possible to overcome this restriction (see Section 5). On the other hand, for reactions with only one reactant as our model of Axin de-/phosphorylation, it is necessary to introduce a dummy communication partner, which we call Timer () throughout the paper. Axin () communicates with Timer() on channel pho and then turns into AxinP (). AxinP() proceeds analogously on channel dep.

A $\pi$-Calculus with stochastic semantics, see (Priami 1995), allows to perform stochastic simulations in the lines of Gillespie (1976). A stochastic semantics requires reactions with rate constants. Since in the $\pi$-Calculus, reactions involve exactly one sender and one receiver, we introduce rate constants by assigning a real number to the receiver. Thus, in our previous example phosphorylated Axin is represented by AxinP ( $) \triangleq \operatorname{dep}\left[k_{d e p}\right]$ ? (). Axin (), where the rate constant of the reaction is given within []. 


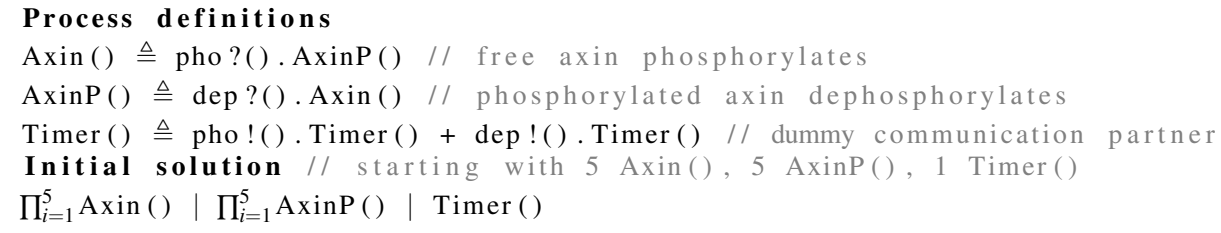

Figure 4: A simple model of Axin de-/phosphorylation in the $\pi$-Calculus

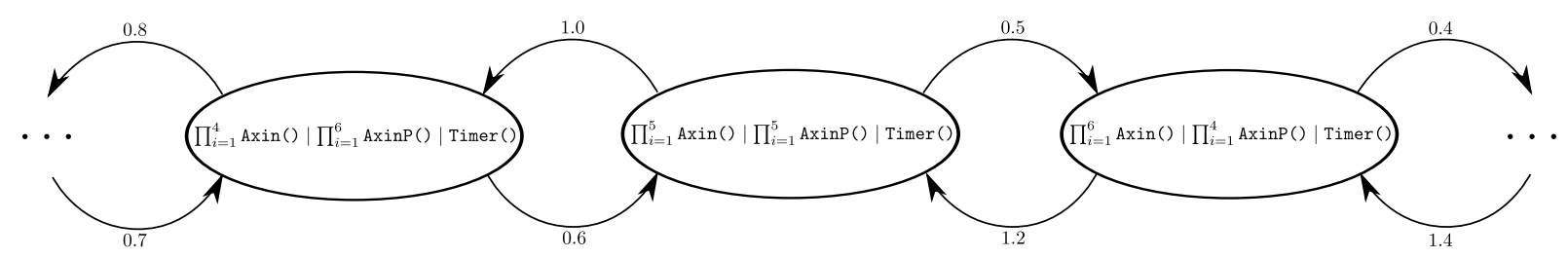

Figure 5: A part of the Continues Time Markov Chain with reactions and initial solution as described in the example

The stochastic semantics of the $\pi$-Calculus defines rules for building a Continues Time Markov Chain (CTMC). Based on the structural congruence of the $\pi$-Calculus, the states of the chain are given by the equivalence classes of all solutions reachable from the initial solution. For example, ( $\left.\prod_{i=1}^{6} \operatorname{Axin}()\left|\prod_{i=1}^{4} \operatorname{AxinP}()\right| \operatorname{Timer}()\right)$ and (Timer() $\mid \prod_{i=1}^{4}$ AxinP () $\mid \prod_{i=1}^{6}$ Axin()) represent one successor state of the initial solution. Transitions between the states are labeled with transition rates. Transition rates are derived by first grouping all reactions that lead to the same state and summing up their rate constants. The initial solution in Figure 4, for example, yields two successor states with transition rates as illustrated in Figure 5, with $k_{p h o}=0.2$ and $k_{d e p}=0.1$. Notice, that by defining the transition rates in this way, we obtain Mass Action as the underlying reaction kinetics. For example, consider the reaction of Axin() to AxinP () with 5 Axin () as in the initial state. According to the law of Mass Action, we need to determine the product of the amounts of the two reactants, i.e. the sender and the receiver, and the rate constant, and thus obtain $1 \cdot 5 \cdot 0.2=1.0$. Notice, that the stochastic semantics of the $\pi$-Calculus provides facilities for assigning infinite rate constants to reactions. Reactions with infinite rate constants happen immediately without time consumption.

The Attribute Language of the Imperative $\pi$-Calculus. We define an attribute language $\mathscr{L}$ that allows to express both attributes and reaction constraints. We keep $\mathscr{L}$ rather abstract, i.e. we define its basic properties but leave it to the user to introduce a concrete instance. This allows for an adaption of the language to the respective application area. We require $\mathscr{L}$ to define a call-by-value $\lambda$-Calculus. This is essential because, as shown above, reaction rules are split into two parts, the sender and the receiver. Therefore, we must be able to split reaction constraints. This is done by defining functions, i.e. $\lambda$-Calculus abstractions, on the receiver side and an argument on the sender side. To determine the rate constant of a communication (reaction) the function on the receiver side is applied to the argument on the sender side.

$\mathscr{L}$ defines a tuple (Consts, Succ, $\Downarrow$ ), where Consts refers to a set of basic constants of the language, like $\mathbb{R}$, Booleans, Strings, or functional constants like $+,-, \star, /$, or $=$. We freely use infix notation. As already mentioned above, the Imperative $\pi$-Calculus introduces a global store that maps (channel) names to values. Thus, Consts must contain the function constant val that returns the value of channel names. Succ represents the set of successful values in Consts, i.e. those values that enable reactions as the result of reaction constraint evaluation. For example, in our introducing examples and also throughout the paper, values in $\mathbb{R}_{>0}$ are successful values. Rate constants different to that, e.g. 0 , do not allow for reactions to occur.

In order to evaluate expressions, we introduce with $\Downarrow$ a big step evaluator, that maps expressions to values. More precisely, $\Downarrow$ is a partial function Exprs $\times$ Env $\rightarrow$ Vals $\times$ Env, where Exprs is a set of expressions, Env a set of environments, and Vals a set of values. With Env, we account for the global store of the Imperative $\pi$-Calculus. In $\mathscr{L}$ values cover the set of constants Consts, the set of channel names Chans, and the $\lambda$-Calculus abstraction $\lambda$ x.e, with $e \in$ Exprs. Depending on the application the set of values in $\mathscr{L}$ can be extended by other constructs, e.g. pairs. The set of expressions Exprs contains besides the usual $\lambda$-Calculus application $e_{1} e_{2}$, with $e_{1}, e_{2} \in$ Exprs, also the assignment $e_{1}:=e_{2}$ to modify the mapping in the global store. In the modeling, we also use conditions of the form if $e$ then $e_{1}$ else $e_{2}$ and sequences $e_{1} ; e_{2}$, which are 


\begin{tabular}{|c|c|c|c|c|}
\hline Processes & $P, Q$ & $::=$ & $\begin{array}{l}A(\tilde{e}) \\
P_{1} \mid P_{2} \\
(v x: e) P \\
\sum \\
\mathbf{0}\end{array}$ & $\begin{array}{l}\text { defined process } \\
\text { parallel composition } \\
\text { channel creation } \\
\text { sums } \\
\text { empty solution }\end{array}$ \\
\hline Sums & & & $\begin{array}{l}\pi . P \\
\Sigma+\Sigma^{\prime}\end{array}$ & $\begin{array}{l}\text { prefixed process } \\
\text { summation }\end{array}$ \\
\hline Prefixes & $\pi$ & & $\begin{array}{l}e_{1}\left[e_{2}\right] ? \tilde{y} \\
e_{1}\left[e_{2}\right] ! \tilde{e}\end{array}$ & $\begin{array}{l}\text { receiver } \\
\text { sender }\end{array}$ \\
\hline Definitions & $D$ & & $A(\tilde{x}) \triangleq P$ & parametric process definition \\
\hline
\end{tabular}

Figure 6: Syntax of the Imperative $\pi$-Calculus: $e, e_{1}, e_{2}, \tilde{e}$ are expressions and $v, \tilde{v}$ values of $\mathscr{L}$, and $x, \tilde{x}, \tilde{y} \in$ Chans

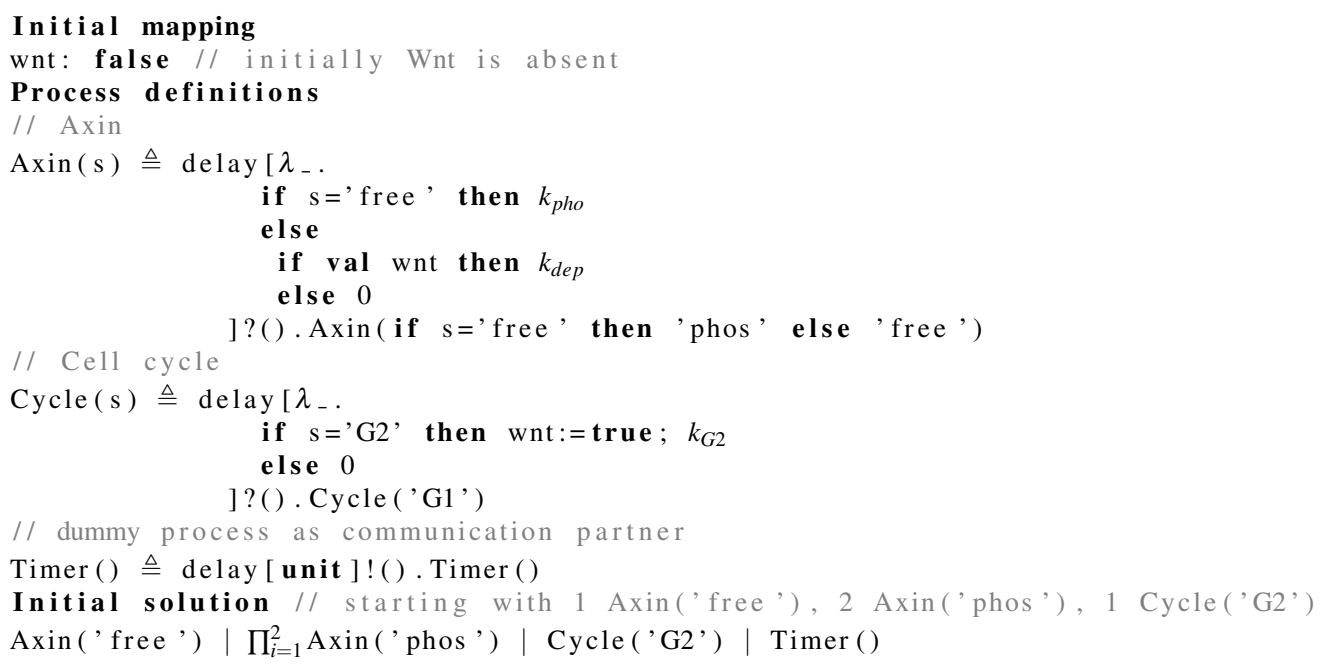

Figure 7: A model of Axin de-/phosphorylation depending on the cell cycle in the Imperative $\pi$-Calculus

basic syntactic sugar. As usual, the value of sequences is given by the evaluation of the second expression.

Obtaining the Imperative $\pi$-Calculus by extending the $\pi$-Calculus. The syntax of the Imperative $\pi$-Calculus can be seen in Figure 6. It extends the $\pi$-Calculus in three ways:

1. Values are assigned to channels.

2. Constraints are associated to communications, which are defined in [] on the sender and receiver side (replacing rate constants).

3. Expressions define the arguments of defined processes, subjects of senders and receivers, and objects of senders.

Since we introduce a global store, programs in the Imperative $\pi$-Calculus contain, besides process definitions and an initial solution, also an initial mapping from names to values. For example, a model describing the reactions in Figure 2 is shown in Figure 7. The constant unit defines a dummy value. The values of names which are not mentioned in the initial mapping are assumed to be unit. Since all reactions have only one reactant, their constraints can be fully declared on the receiver side. Thus, the abstractions are defined without any parameter $\left(\lambda_{-}\right)$and the senders provide unit as arguments.

The stochastic semantics of the Imperative $\pi$-Calculus comes with two major differences: First, the states of the CTMC are not only defined by the processes in the solutions but also by the mapping in the global store. Thus in our example above, (Axin('free') $\mid \prod_{i=1}^{2}$ Axin('phos') |Cycle()| Timer(), wnt: false) represents a different state than 


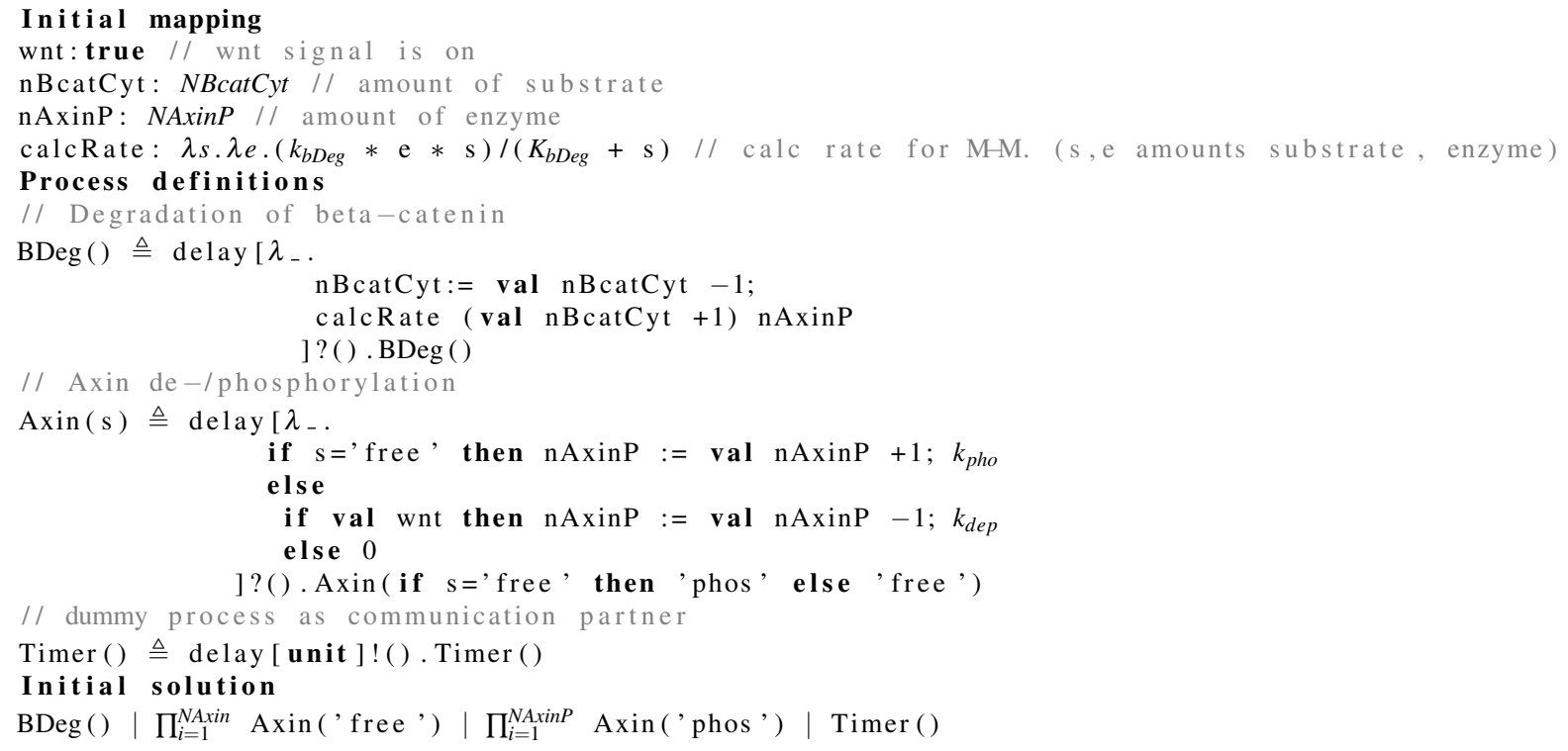

Figure 8: Implementation of Axin mediated $\beta$-catenin degradation with with Michaelis-Menten kinetics in combination with Axin de-/phosphorylation in the Imperative $\pi$-Calculus

(Axin ('free') | $\prod_{i=1}^{2}$ Axin ('phos') | Cycle() | Timer(), wht:true). Second, before calculating transition rates, the rate constants of all reactions are determined by first evaluating the constraint expressions of all senders and receivers separately and then, for each reaction, applying the abstraction of the receiver to the argument of the sender. Consecutively, only those reactions are taken into account whose constraints evaluate to successful values.

\section{IMPLEMENTING MICHAELIS-MENTEN KINETICS IN THE IMPERATIVE $\pi$-CALCULUS}

As described in Section 3, the rate equation for Axin mediated $\beta$-catenin degradation yields $\left(k_{b D e g} \cdot[\right.$ AxinP $\left.] \cdot[B c a t C y t]\right) /\left(K_{b D e g}+\right.$ $[$ BcatCyt $])$. The law of Mass Action is hard wired to the stochastic semantics of the $\pi$-Calculus. Thus, although only dependent on the amounts of two reactants, reactions with Michaelis-Menten kinetics cannot be implemented in a senderreceiver manner. However, it is possible to bypass Mass Action kinetics by setting the amounts of the sender and the receiver to one. By this means, reaction rates entirely depend on their rate constants, which are then defined to represent the intended Michaelis-Menten kinetics. Thus, for an appropriate encoding it must be possible for the modeler to trace the amounts of substrate and enzyme and calculate rate constants depending on them. Notice, that with every change of the substrate or enzyme amounts, which can be caused by more than one reaction, corresponding rate constants need to be updated.

As an example for an encoding of Michaelis-Menten kinetics in the Imperative $\pi$-Calculus, we provide an implementation of Axin mediated $\beta$-catenin degradation in combination with Axin de-/phosphorylation in Figure 8. Our approach bases on the idea of rate constants that depend on values in the global store. Thus, we introduce two channels, nBcatCyt and nAxinP, that refer to the amounts of the substrate and the enzyme. They are initialized with the initial amounts of $\beta$-catenin in the cytosol (NBcatCyt) and phosphorylated Axin (NAxinP). $\beta$-catenin degradation is represented by BDeg (). As a side effect BDeg () decreases the amount of $\beta$-catenin in the cytosol when communicating with Timer ( ). By applying function calcRate the rate constant is obtained according to Michaelis-Menten kinetics. Notice, that due to sequence evaluation, the side effect needs to be performed before determining the rate constant. This requires to apply calcRate to the amount of $\beta$-catenin in the cytosol $n B$ cat $C y t$ increased by one. The implementation of Axin de-/phosphorylation extends on the one in Figure 7 only slightly by updating the amount of phosphorylated Axin (nAxinP) accordingly. Because of constraint evaluation, this has immediate effect on the rate constant of $\beta$-catenin degradation. For the sake of simplicity, we omit the cell cycle process.

We see this kind of implementation rather as a workaround. Ultimately, solutions have to be found for a stochastic semantics that allows to take different kinetics into account. However, the encoding is interesting beyond Michaelis-Menten kinetics, as it is neither limited in the number of reactants nor fixed to some specific kinetics. 
Our implementation of Michaelis-Menten kinetics does not translate to the $\pi$-Calculus, because there rate constants are statically assigned to reactions. Thus, it would be necessary to enumerate the reactions for all possible, possibly infinitely many, combinations of the amounts of phosphorylated Axin and $\beta$-catenin in the cytosol. Even in the bounded case, an implementation is hard to obtain, as those reactions that share reactants affect each other, which requires communication protocols based on infinite rate constants for immediate updates. Modeling tools that base on the $\pi$-Calculus (Priami et al. 2001, Kuttler et al. 2007) provide mechanisms to trace the amount of reactants and define reaction rates depending on them, which already points to the need of an imperative extension. However, as they are clearly not part of the formal language, we do not regard these approaches here.

\section{CONCLUSION AND OUTLOOK}

Based on a model of the $\mathrm{Wnt} / \beta$-catenin signaling pathway, we provided a case study on integrating heterogeneous data stemming from an existing model, the literature, and wet-lab experiments. In particular, we showed how to obtain model parameters, e.g. diffusion and reaction rate constants, and also Michaelis-Menten rate equations for stochastic models. In order to explore its expressiveness, we used the Imperative $\pi$-Calculus as our modeling language. In this context, we provided, to the best of our knowledge, the first implementation of a reaction with Michaelis-Menten kinetics in a $\pi$-Calculus based approach. Our future work is following two major directions. In order to investigate the influence of the cell cycle on the activity of the Wnt signaling pathway, we want to fit the behavior of our model to the one presented in Lee et al. (2003) and thoroughly analyze its behavior. Then, our goal is to not just consider single cells but entire populations producing and propagating Wnt molecules. In this refined multi-cell model our next steps will be to consider processes of division and differentiation explicitly. On the computer science side, we will continue to explore the generality of our implementation of Michaelis-Menten kinetics in the Imperative $\pi$-Calculus according to the encoding of reactions with more than two reactions and different kinetics.

\section{ACKNOWLEDGMENTS}

We thank Matthias Jeschke for his help on the calculation of diffusion constants, Anne Schmoele and Jana Frahm for their help with cell cycle experiments. This work is financed by the Deutsche Forschungsgemeinschaft (DFG) as part of the research training school dIEM oSiRiS, and the research grant dIErMoSiS.

\section{REFERENCES}

Alam, S., A. Sen, L. A. Behie, and M. Kallos. 2004. Cell Cycle Kinetics of Expanding Populations of Neural Stem and Progenitor Cells In Vitro. Biotechnology and Bioengineering 88:332-347.

Breitling, R., D. Gilbert, M. Heiner, and R. Orton. 2008. A Structured Approach for the Engineering of Biochemical Network Models, Illustrated for Signalling Pathways. Briefings in Bioinformatics 9 (5): 404-421.

Cho, K., S. Baek, and M. Sung. 2006. Wnt Pathway Mutations Selected by Optimal Beta-Catenin Signaling for Tumorigenesis. FEBS Letters 580:3665-3670.

Ciocchetta, F., and J. Hillston. 2008. Bio-PEPA: An Extension of the Process Algebra PEPA for Biochemical Networks. ENTCS 194 (3): 103-117.

Ciocchetta, F., J. Hillston, M. Kos, and D. Tollervey. 2008. Modelling Co-Transcriptional Cleavage in the Synthesis of Yeast Pre-rRNA. TCS 408 (1): 41-54.

Danos, V., J. Feret, W. Fontana, R. Harmer, and J. Krivine. 2007. Rule-Based Modelling of Cellular Signalling. In International Conference on Concurrency Theory (CONCUR), ed. L. Caires and V. Vasconcelos, Volume 4703 of LNCS, 17-41: Springer.

Elf, J., and M. Ehrenberg. 2004. Spontaneous Separation of Bi-Stable Biochemical Systems into Spatial Domains of Opposite Phases. Systems Biology, IEE Proceedings 1 (2): 230-236.

Gillespie, D. T. 1976, December. A General Method for Numerically Simulating the Stochastic Time Evolution of Coupled Chemical Reactions. Journal of Computational Physics 22 (4): 403-434.

Heiner, M., R. Richter, M. Schwarick, and C. Rohr. 2008. Snoopy - A Tool to Design and Execute Graph-Based Formalisms. Petri Net Newsletter 74:8-22.

John, M., C. Lhoussaine, and J. Niehren. 2009. Dynamic Compartments in the Imperative Pi Calculus. In Computational Methods in Systems Biology, 7th International Conference, ed. P. Degano and R. Gorrieri: Springer. to appear.

John, M., C. Lhoussaine, J. Niehren, and A. Uhrmacher. 2008. The Attributed Pi Calculus. In Computational Methods in Systems Biology, 6th International Conference, Volume 5307, 83-102: Springer. 
Krieghoff, E. I. 2006. Analyzing the Regulation of the Subcellular Localization of beta-catenin by Fluorescence Recovery After Photobleaching. Ph. D. thesis, Friedrich-Alexander-Universitt Erlangen-Nrnberg.

Kuttler, C., C. Lhoussaine, and J. Niehren. 2007. A Stochastic Pi Calculus for Concurrent Objects. In Second International Conference on Algebraic Biology, ed. H. Anai, K. Horimoto, and T. Kutsia, Volume 4545 of LNCS, 232-246: Springer.

Kuttler, C., and J. Niehren. 2006. Gene Regulation in the Pi Calculus: Simulating Cooperativity at the Lambda Switch. TCSB 4230/2006:24-55.

Lange, C., E. Mix, K. Rateitschak, and A. Rolfs. 2006. Wnt Signal Pathways and Neural Stem Cell Differentiation. Neurodegenerative Diseases 3 (1-2): 76-86.

Lecca, P., C. Priami, P. Quaglia, B. Rossi, C. Laudanna, and G. Constantin. 2004. A Stochastic Process Algebra Approach to Simulation of Autoreactive Lymphocyte Recruitment. SIMULATION 80 (6): 273-288.

Lee, E., A. Salic, R. Krüger, R. Heinrich, and M. Kirschner. 2003. The roles of APC and Axin derived from experimental and theoretical analysis of the Wnt pathway. PLoS Biol 1 (1): 116-132.

Milner, R. 1999. Communicating and mobile systems: the $\pi$-calculus. Cambridge University Press.

Panté, N., and M. Kann. 2002. Nuclear Pore Complex Is Able to Transport Macromolecules with Diameters of $39 \mathrm{~nm}$. Molecular Biology of the Cell 13:425-434.

Pollock, K., P. Stroemer, S. Patel, L. Stevanato, A. Hope, E. Miljan, Z. Dong, H. Hodges, J. Price, and J. Sinden. 2006. A Conditionally Immortal Clonal Stem Cell Line from Human Cortical Neuroepithelium for the Treatment of Ischemic Stroke. Experimental Neurology 199(1):989-995.

Priami, C. 1995. Stochastic $\pi$-Calculus. Computer Journal 6:578-589.

Priami, C., A. Regev, E. Shapiro, and W. Silverman. 2001. Application of a Stochastic Name-Passing Calculus to Representation and Simulation of Molecular Processes. Information Processing Letters 80:25-31.

Regev, A., and E. Shapiro. 2004. The Pi Calculus as an Abstraction for Biomolecular Systems. In Modelling in Molecular Biology, ed. G. Ciobanu and G. Rozenberg, 219-266. Springer.

Schaeffer, O. 2008. On the Use of Process Alegbra Techniques in Computational Modelling of Cancer Initiation ans Development. Ph. D. thesis, School of Computer Science University of Birmingham.

Smith, J. A., and L. Martin. 1973. Do Cells Cycle ? Proceedings of the National Academy of Sciences of the United States of America 70(4):1263-67.

Tymchyshyn, O., and M. Kwiatkowska. 2008. Combining Intra- and Inter-cellular Dynamics to Investigate Intestinal Homeostasis. In Formal Methods in Systems Biology: First International Workshop, FMSB 2008, Cambridge, UK, June 4-5, 2008, Proceedings, 63-76. Springer.

Willert, K., and K. Jones. 2006. Wnt signaling: is the party in the nucleus? Genes Development 20:1394-1404.

Wolkenhauer, O., M. Ullah, W. Kolch, and K. H. Cho. 2004. Modelling and Simulation of IntraCellular Dynamics: Choosing an Appropriate Framework. IEEE T Nanobiosci 3 (3): 200-207.

\section{AUTHOR BIOGRAPHIES}

ORIANNE MAZEMONDET holds a MSc in Bioinformatics and is currently a PhD student of the graduate school dIEM oSiRiS <http://www.diemosiris.de>.

MATHIAS JOHN holds a MSc in Computer Science and is currently a PhD student of the graduate school dIEM oSiRiS $<$ http://www.diemosiris.de>.

CARSTEN MAUS holds a MSc in Biology and is currently a PhD student of the graduate school dIEM oSiRiS $<$ http://www.diemosiris.de>.

ADELINDE M. UHRMACHER is an Associate Professor at the Department of Computer Science at the University of Rostock and head of the Modeling and Simulation Group <http://wwwmosi.informatik.uni-rostock. de>.

ARNDT ROLFS is head of the Albrecht-Kossel Institute <http://www. neurobiology-lab. de $>$. 